\title{
Role of Diffusion Weighted Magnetic Resonance Imaging (DW- MRI) and Apparent Diffusion Coefficient (ADC) in Evaluation of Urinary Bladder Cancer
}

\author{
Mohamed Abd El-Baset ${ }^{a}$, Ahmed F. Yousef ${ }^{a}$, Tarek Abd Elmoneim El-Diasty ${ }^{b}$ \\ a Department of radiology \\ Benha faculty of medicine, \\ Benha University, Egypt. \\ b Department of radiology, \\ urology and nephrology center, \\ Mansoura university, Egypt. \\ Correspondence to: \\ Mohamed Abd El-Baset, \\ Department of radiology \\ Benha faculty of medicine, \\ Benha University, Egypt. \\ Email: \\ dr.m.refaat@hotmail.com \\ Received: 1 February 2020 \\ Accepted:1 March 2020

\section{Abstract:} \\ Background: Urinary bladder cancer $(\mathrm{BC})$ is reported as the $2^{\text {nd }}$ \\ most common genitourinary cancer in males and the $9^{\text {th }}$ most \\ common in females. Accurate pre-surgical evaluation of the local \\ stage of the bladder cancer is crucial in the treatment strategy. So it \\ is very important to evaluate and no invasive imaging modality to \\ help accurate staging of the disease. Purpose: To evaluate role of \\ diffusion weighted MR imaging (DW-MRI) in detection, T-staging \\ of urinary bladder carcinoma. Patients and methods: During the \\ period between November 2018 and November 2019, 44 patients \\ (36 males and 8 females) presenting with haematuria, lower urinary \\ symptoms and urinary bladder mass detected by ultrasound, were \\ enrolled prospectively. All patients underwent standard MRI \\ protocol and diffusion weighted MR imaging of urinary followed by \\ transurethral cystoscopy. Then transurethral resection of the bladder \\ tumor (TURBT) or radical cystectomy according to the tumor \\ staging with histological examination of resected specimens. Results: Correlation between MR \\ and cystoscopy showing $100 \%$ sensitivity in detection of the lesions and as regard to MRI \\ staging of bladder carcinoma and histopathological results the study revealed that 38 patients \\ (86.3\%) had the same T stage while 6 patients (13.6\%) were overstaged. Conclusion: DWI have \\ very high sensitivity in detection of the bladder lesions with accurate predictions of the $\mathrm{T}$ staging \\ of bladder carcinoma can be made by means of DWI.
}

Keywords: Diffusion weighted image, urinary bladder cancer, staging, DWI 


\section{Introduction}

Urinary bladder cancer (BC) was reported as the second most common genitourinary malignancy among males and as the ninth most common among females in the United States (1). Distinguishing between superficial tumors (stage $\leq \mathrm{T} 1)$ and invasive tumors (stage $\geq \mathrm{T} 2$ ) is a corner stone in management of urinary bladder tumors, because each category is managed by different methods, as transurethral resection with or without intravesical chemotherapy for the first group and radical cystectomy, radiation therapy, chemotherapy, or a combination for the second group. So, it will be very important if preoperative imaging could precisely differentiate between both of these groups of bladder cancer. (2)

The contrast-enhanced computed tomography (CT) causes underestimation of the disease in up to $40 \%$ of cases, and it was previously reported that it can only marginally discriminate tumor stages Ta to T3a. (3)

In the recent years the applications of DWI in oncologic area is growing after new advances in the technical points of acquisition and administration of high magnetic field MRI machines. (4)

2. The aim of the study: this study aims to evaluate the value of diffusion weighted imaging (DWI) in detection and T-staging of urinary bladder cancer.

\section{Patients and methods}

Population

Between November 2018 and November 2019, $44 \quad$ patients of urinary bladder malignant lesions were prospective enrolled. They were complaining of haematuria and symptoms suggestive of bladder cancer and were referred for pelvic MRI, after ultrasound detection of urinary bladder mass. The study was done in urology and nephrology centre, Mansoura university.

Inclusion criteria : were patients who: (a) had a history of symptoms consistent with lower urinary tract symptoms, such as haematuria. (b) Had recent ultrasound examination confirming urinary bladder mass. (c) Available final histopathological report of the patient after TURBT or radical cystectomy.

Exclusion criteria: were the common contraindications to MRI (pacemaker, 
metallic foreign bodies, and impaired renal function) or unfit for surgery.

This study was approved by the ethics committee of our institution; an informed consent was obtained from all patients after full explanation of the benefits and risks of the procedure.

\section{Methods}

3-T MRI scanner (Phillips, ingenia 3T, The Netherlands) with 18-channel phased external array coil. Patients did not undergo bowel preparation, but were instructed to fast for at least $4 \mathrm{~h}$ before the exam. The standard position for pelvic MRI is the comfortable supine position with a bolster placed under the knees; $20 \mathrm{mg}$ of hyoscineNbutylbromide (Buscopan) was injected 30 min before examination to reduce intestinal peristalsis.

Pulse sequences and scanning planes

For all patients the imaging protocols consisted of using multi sections spin echo sequences with different repetition time (TR) and echo delay time (TE) to obtain:

1- T2-weighted imaging:

Three planes of multiplanar (axial, coronal, and sagittal) $\mathrm{T} 2 \mathrm{~W}$ images without fat suppression are obtained with TR (ms) 4690, TE (ms) 119, Flip angle (degree) 90, FOV (cm) 23, Matrix 400x256-302, Slice thickness (mm) 3-4, Slice gap (mm) 0-0.4 \& Number of excitations 2-3.

2- Diffusion-weighted imaging: was performed during free breathing with axial and coronal planes fat suppressed water-excited single-shot spin echo with high b value (800-1000 s/mm2) TR (ms) 2500 up to 5300, TE (ms) 61, Flip angle (degree) 90, FOV (cm) 32, Matrix 128x128, Slice thickness (mm) 3-4, Slice gap (mm) 0.3-0.4 \& Number of excitations 4-10.

After MRI, cystoscopy was done for biopsy and confirming malignancy. All suspicious papillary lesions 21/44 patients underwent transurethral resection and intravesical BCG chemotherapy for malignancy proved lesions, while 23/44 patients underwent radical cystectomy and radiotherapy.

\section{Image analysis}

The tumor margin, integrity of the underlying hypointense line of the bladder wall and degree of extent into the perivesical fat were assessed based on the appearance of tumor on T2-weighted imaging, then augmented by the diffusion weighted image with using integrated images. The mean ADC values of the lesions were measured. The radiologist was blinded to the results of cystoscopy. 
Statistical analysis

Comparison between the reported MRI staging and final histopathological staging. The IBM SPSS Corp. (Version 22.0. Armonk, NY: IBM Corp) was used.

\section{Results}

As regard to the MRI T-Staging of 44 cases (using T2WI and DWI) there were 22 patients at stage $\mathrm{T} 1,11$ patients at stage $\mathrm{T} 2$, 15 patients at stageT3 and 2 patients at stage T4. These results were compared with final histological reports of the taken specimens. 21/44 patients underwent Trans-urethral resection of the bladder tumor (TURBT), while 23/44 patients underwent radical cystectomy.

On comparison between MR findings \& histopathological findings, we found 38/44 (86.4\%) patients had the same $\mathrm{T}$ stage (Figs. 1), 6/44 (13.6 \%) patients were overstaged. Regarding the overstaged patients, the pathological report of 1 of them proved that the tumor is only invading the muscle layer (stage 2) while MRI report showed that there was macroscopic focal invasion of the perivesical fat (stage $3 b$ ). In another 4 overstaged patients, pathological reports revealed that tumor invading the lamina propria only (stage 1) while MRI report proved invasion of the muscle layer in addition to lamina propria (stage 2). And the last overs taged case was pathological proved that the tumor is invading the perivesical fat with no invasion of the nearby organs (stage 3b) while MRI report showed that there was focal invasion of the seminal vesicles (stage $4 a$ ) in addition to the fat invasion.

As regard to the value of using DWI with the T2WI in staging of the bladder masses, it was found that the sensitivity in detection of the lesions was $100 \%$ even with the very small lesions. Regarding the differentiation between superficial and invasive tumor (chart 1) it shows specificity $80 \%$, accuracy $90.9 \%$, PPV $85.7 \%$ and NPV $100 \%$ ( $p$ $<0.001)$ (table 1).

Regarding the individual stage differentiation, on differentiation between $\mathrm{T} 1 \& \mathrm{~T} 2$ stages we found specificity $80 \%$, accuracy 84\%, PPV 55.6\% and NPV $100 \%$ $(p=0.002)$, while on differentiating T2 from T3b we found specificity $83.3 \%$, accuracy 93.3\%, PPV 90\% and NPV 100\% ( $p=$ $0.002)$.

The diffusion weighted imaging revealed that all carcinomas were restricted in diffusion. The mean and standard deviations of the ADC values ( $\mathrm{x} 10^{-3} \mathrm{~mm}^{2} / \mathrm{s}$ ) were $0.91 \pm 0.28$. 

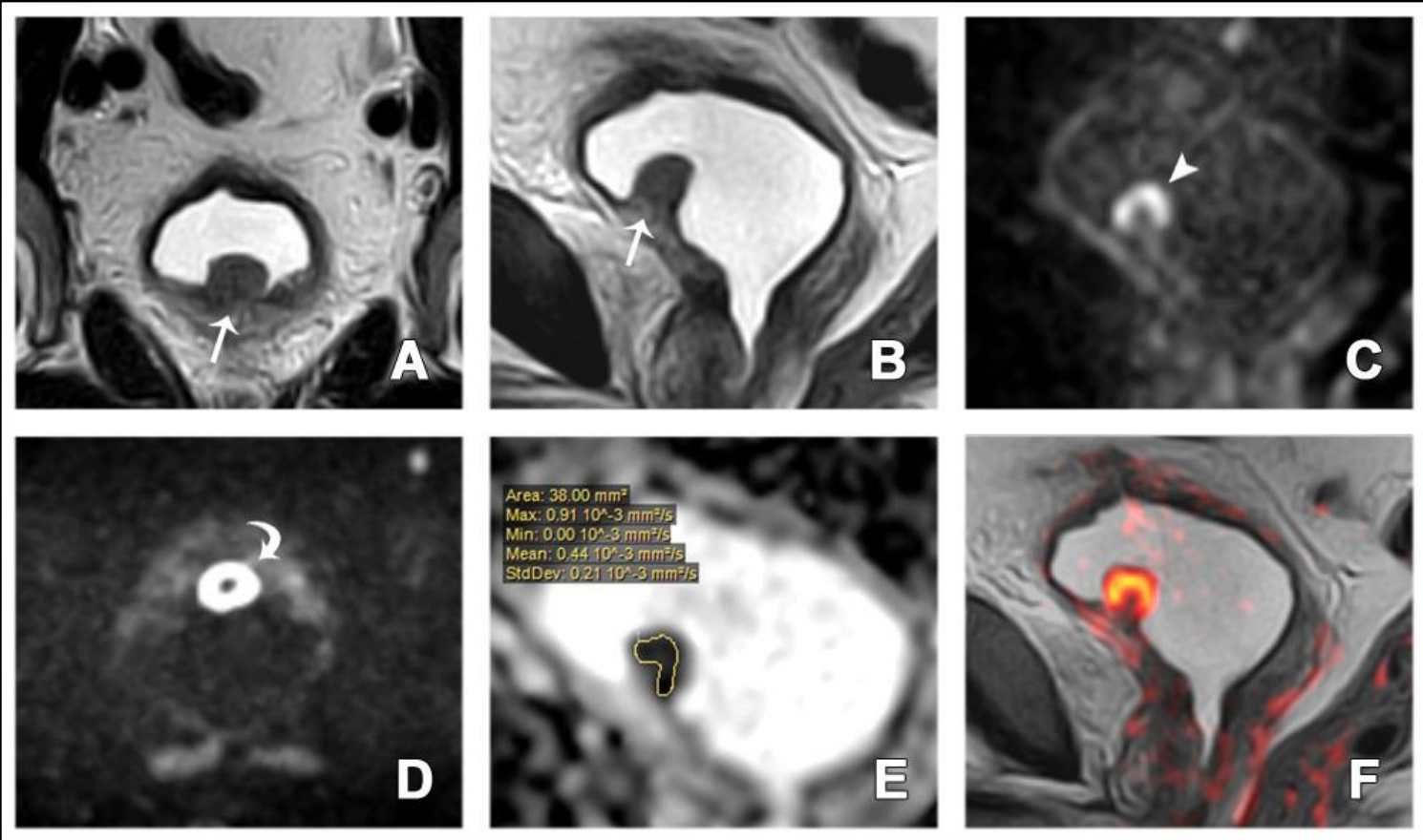

Figure 1: Patient with superficial papillary urothelial carcinoma of GIII and T1 stage. (A) coronal and (B) sagittal high resolution T2WI show polypoidal soft tissue mass arising from the anterior wall of the bladder with the hypointense line of the muscle layer at its base seems to be interrupted (white arrow), suggesting invasion of the muscularis propria. (C) sagittal DWI in the plane of the lesion showing superficial hyperintensity of the lesion (white arrow head) with well-defined hypointense central stalk inside it giving "inch worm" sign, suggestive of superficial papillary nature (D) reformatted axial DWI on the bladder showing transverse section in the lesion (curved arrow) with peripheral hyperintensity and central hypointense central stalk. (E) sagittal ADC map showing hypointensity of the lesion confirm diffusion restriction with the mean ADC value is $0.44 \times 10^{-3} \mathrm{~mm}^{2} / \mathrm{s}$. (F) sagittal integrated overlay color-coded view of the T2WI and DWI demonstrating more anatomical and functional data, and confirming its papillary superficial nature.

Table (1): Statistical evaluation of the role of MRI in differentiating superficial from invasive tumor.

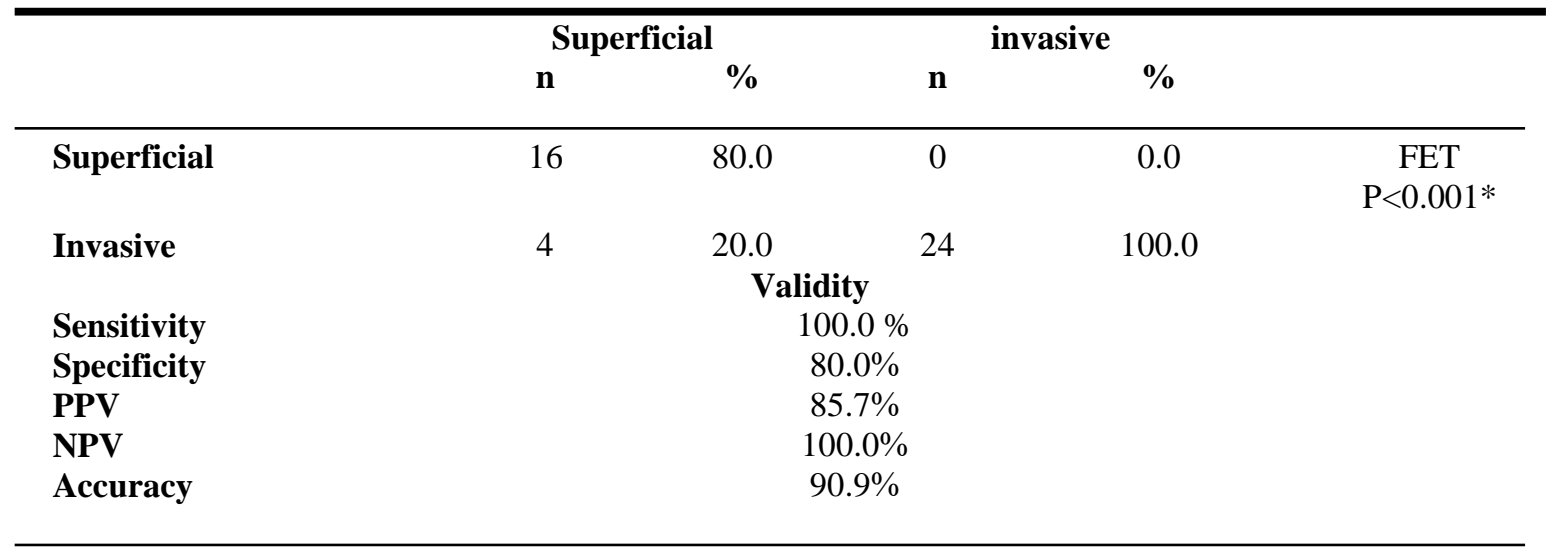




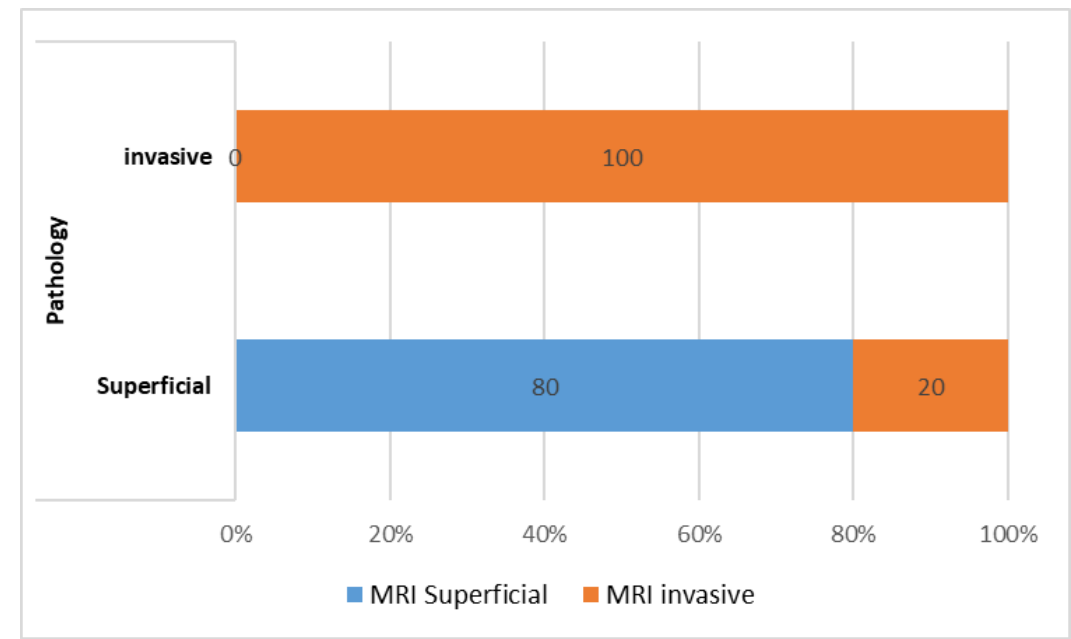

Chart (1): Demonstrating value of T2WI \& DWI in differentiating the superficial from muscle invasive lesions as compared to the final pathological staging.

\section{Discussion}

On viewing T2WI of 44 patients with bladder carcinoma, the musculosa propria of the bladder wall appeared as a hypointense line. In patients with superficial or papillary tumors, this layer was intact without interruption. In patients with muscleinvasive tumors, the hypointensity of this muscle was interrupted by the tumor which is of less hypointensity degree. Yet in some patient the hypointense muscle layer appears to be thickened and partially deformed making it difficult to be sure about the degree of invasion, but with aid of the DWI which clearly demonstrate the deep surface of the tumor differentiated from the related fibrotic and inflammatory changes it becomes easier to evaluate the degree of invasion. The same in patients with extravesical disease spread, DWI was very

helpful in differentiation between organconfined versus non-organ-confined disease. With using the integrated images with overviewing the DWI as colour encoded images over the T2WI giving better demonstration of the functional date with anatomical data.

By correlation between the results staging the bladder carcinomas using DWI and T2WI and histopathological results we found that the overall accuracy was $86.4 \%$, 38/44 patients had the same $\mathrm{T}$ stage, 6/44 patients were overstaged. Our result is close to that reported by others having their overall accuracy $88 \%$. (5) 
As regard to differentiating between $\mathrm{T} 1$ or lower tumors (non-invasive) and $\mathrm{T} 2$ or higher (invasive) tumors our results was in harmony with the results reported by Takeuchi et al. to be of accuracy 96\%, sensitivity $100 \%$ and specificity $88 \%$ (5) while other studies had reported $63.6 \%$ accuracy for DWI alone and $6.1 \%$ for T2WI alone. (6)

Regarding differentiation between $\mathrm{T} 2$ or less (organo-confined) and T3 or more (nonorgano-confined) we found that both the T2WI \& DWI together shows higher accuracy as compared to a study done where it was reported that the accuracy was $69.6 \%$ for DWI alone and $15.1 \%$ for T2WI alone.

\section{Conclusion}

Adding DWI and ADC quantification to the routine conventional MRI evaluation of the bladder carcinomas increase the accuracy of local staging of the lesion and differentiating superficial lesions from muscle invasive lesions.

To cite this article: Mohamed Abd El-Baset, Ahmed F. Yo
Role of Diffusion Weighted Magnetic Resonance Imaging
Coefficient (ADC) in Evaluation of Urinary Bladder Canc
DOI: $10.21608 / \mathrm{bmfj} .2021 .24956 .1219$

Biomarker for Bladder Cancer. American Journal of Roentgenology. 2017;208(6):1218-28.

2. Dighe MK, Bhargava $\mathrm{P}$ and Wright $\mathrm{J}$. Urinary bladder masses: techniques, imaging spectrum, and staging. Journal of computer assisted tomography. 2011;35(4):411-24.

3. Maurer T, Horn T, Heck M, Gschwend JE, Eiber M, Beer AJ. Current Staging Procedures in Urinary Bladder Cancer. Diagnostics (Basel). 2013;3(3):315-24.

4. Sherif MF. The value of diffusion weighted MR imaging in $\mathrm{T}$ staging and correlation with histologic grading in urinary bladder cancer. The Egyptian Journal of Radiology and Nuclear Medicine. 2015;46(1):189-94.

5. Takeuchi M, Sasaki S, Ito M, Okada S, Takahashi S, Kawai T, et al. Urinary Bladder Cancer: Diffusion-weighted MR Imaging-Accuracy for Diagnosing T Stage and Estimating Histologic Grade. 2009;251(1):112-21.

6. El-Assmy A, Abou-El-Ghar ME, Mosbah A, El-Nahas AR, Refaie HF, Hekal IA, et al. Bladder tumour staging: comparison of diffusion- and T2weighted MR imaging. European radiology. 2009;19(7):1575-81. 